Check for updates

Cite this: RSC Adv., 2019, 9, 37846

Received 7th August 2019

Accepted 11th November 2019

DOI: $10.1039 / \mathrm{c} 9 \mathrm{ra0} 0806 \mathrm{~g}$

rsc.li/rsc-advances

\section{Eutectic liquid crystal mixture E7 in nanoporous alumina. Effects of confinement on the thermal and concentration fluctuations $\uparrow$}

\author{
Aristoula Selevou, ${ }^{a}$ George Papamokos, ${ }^{a}$ Tolga Yildirim, ${ }^{c}$ Hatice Duran, (D) ${ }^{c}$ \\ Martin Steinhart (D) ${ }^{d}$ and George Floudas (D) *ab
}

\begin{abstract}
The eutectic mixture of liquid crystals E7 is studied in confinement by means of thermal and dielectric measurements. The uniform 1-D confinement provided by self-ordered nanoporous alumina leads to a decrease in the nematic to isotropic transition temperature due to interaction with the pore surface, e.g. surface anchoring. The prevalence of certain dynamic modes of relaxation is found to depend on the surface properties of the confining pores. The dynamics (i.e., relaxation times) were found to accelerate with increasing confinement, resulting in a decreasing glass temperature, independent of surface treatment. From the pre- and meta-transitional dependence of the dielectric permittivity on temperature we are able to deduce a weakening effect of confinement on the nematic to isotropic (N/I) transition which allows the determination of a critical pore diameter (in the range from $11 \mathrm{~nm}$ to $23 \mathrm{~nm}$ ) below which the transition becomes continuous. Comparison of the N/I transition of E7 to those of its constituent liquid crystals reveals a significantly weaker transition occurring over a widened temperature range. This suggest the importance of concentration fluctuations in rounding first order phase transitions that are triggered by the different length scales and ranges of nematic stability in E7. The results have an impact beyond the present case and for several soft materials (e.g. oligomers used as OLEDs, polymers, colloids) as it demonstrates the importance of concentration fluctuations in addition to thermal fluctuation on the strength of phase transitions.
\end{abstract}

\section{Introduction}

The physics of confined soft matter has been extensively studied for a wide array of materials and confining hosts. Rod-like liquid crystals (LCs) are of interest due to their utilization in device fabrication. However, as a significant portion of LCs in technological applications are in contact with hard interfaces, ${ }^{\mathbf{1 , 2}}$ it is necessary to investigate the effects of geometric confinement on the thermodynamics and dynamics of LCs. Apart from size effects, LCs in confined geometries ${ }^{3-6}$ are susceptible to surface interactions such as anchoring and density modulations. ${ }^{7,8}$ Therefore, the director field configuration and, macroscopically, the material's optical and dielectric properties, are

${ }^{a}$ Department of Physics, University of Ioannina, P. O. Box 1186, 45110 Ioannina, Greece.E-mail: gfloudas@uoi.gr

${ }^{b}$ University Research Center of Ioannina (URCI), Institute of Materials Science and Computing, Ioannina, Greece

'Department of Materials Science \& Nanotechnology Engineering, TOBB University of Economics and Technology, Söğ̈̈tözü Cad. 43, Ankara, 06560, Turkey

${ }^{d}$ Institut für Chemie Neuer Materialien, Universität Osnabrück, D-49069 Osnabrück, Germany

$\dagger$ Electronic supplementary information (ESI) available: XPS high-resolution spectra for pristine and ODPA modified AAO. Cartesian coordinates of optimized structures in DFT calculations. See DOI: 10.1039/c9ra08806g directly related to the size of the system as well as the competition of surface effects with the intrinsic elastic forces.

E7, in particular, is a liquid crystalline mixture comprised of four hematogenic compounds with non-coinciding nematic ranges. Its usefulness arises from a long range $(\sim 125 \mathrm{~K})$ of nematic stability at temperatures typical for applications. As such, the literature on E7 is widely concerned with composites with other systems, such as polymer dispersions and ferromagnetic nanoparticles. Bulk dynamics of pure E7 have been studied mainly by dielectric spectroscopy exhibiting various modes of dipolar relaxation. ${ }^{9-16}$ This has also been supplemented by specific heat, ${ }^{9}$ rheological ${ }^{\mathbf{1 0}}$ and neutron scattering ${ }^{\mathbf{1 1}}$ measurements which allow the identification of the molecular process responsible for the dynamics. With respect to the nematic to isotropic (N/I) transition, optical birefringence studies $^{\mathbf{1 2}}$ have deduced a tricritical dependence of the nematic order parameter on temperature. Furthermore, studies on E7 in the presence of nanometric sized colloidal dispersions ${ }^{13}$ and a cellulose derivative ${ }^{14}$ indicated surface induced orientation effects which strengthen the high frequency relaxation mode. In confinement, previous dielectric studies of E7 employed, as confining matrices, either Anopore membranes ${ }^{\mathbf{1 5}}$ or SBA-type molecular sieves $(20 \mathrm{~nm}$ in diameter $){ }^{\mathbf{1 6}}$ In both hosts, a hindered surface relaxation emerged in addition to the bulk- 
like modes and confinement impacted the high frequency mode two fold: first, with an acceleration of dynamics and, second, with an increase in fragility as the system approached its glass temperature. However, already in the bulk state, a satisfactory assignment of all exhibited relaxation modes has not been accomplished. In addition, the previously employed confining geometries lacked the uniformity and full range of diameters afforded by self-ordered nanoporous alumina (AAO). ${ }^{17-20}$ This is necessary if we intend on distinguishing size effects from interactions with a uniform host as well as consistently examining the effect of surface interactions. Lastly, as E7 is a model nematic system, it provides a suitable candidate to examine the impact of confinement on the order of a phase transition (that is clearly of the first kind in the bulk) with implications to the nature of phase transitions in confined soft matter, in general.

In the present work, we investigate the effect of confinement on the thermodynamics and dynamics of the LC mixture E7. Specifically, thermal measurements are used to statically map the temperature ranges of phases exhibited in confinement compared to those in the bulk. Broadband dielectric spectroscopy is employed in the detection of such ranges dynamically and the study of molecular dynamics in confinement, for varying surface conditions. We report a depression and broadening of transition temperatures as well as increased molecular mobility with confinement. Dielectric measurements provide evidence for a weaker N/I transition with decreasing pore size allowing the estimation of a critical pore size below which the transition becomes continuous. Furthermore, a comparison of the N/I transition in E7 and similar alkylcyanobiphenyl molecules indicates a more complex description of the transition for a mixed system as compared to one-component system.

\section{Experimental section}

\section{Materials and method of infiltration}

The investigated liquid crystal was the eutectic mixture E7 (Merck). Self-ordered nanoporous alumina membranes (pore diameters: 25, 35, 65, 200, $400 \mathrm{~nm}$; pore depth $100 \mu \mathrm{m}$ ) were prepared by following the anodization procedures in the literature. ${ }^{17-19}$ Prior to infiltration, AAO membranes were annealed overnight in a vacuum at $443 \mathrm{~K}$. Infiltration was performed, within the material's isotropic phase at atmospheric pressure, at $343 \mathrm{~K}$ and proceeded via capillary action for no more than 6 hours. Following this procedure, samples were brought to ambient temperature and subsequently quenched with liquid nitrogen, and excess E7 was removed from the membrane surfaces with sharp razor blades and soft nonabrasive polishing paper. The difference in membrane mass before and after infiltration is calculated as the mass of the infiltrated sample. Typically, the sample mass was $\sim 12 \mathrm{mg}(400 \mathrm{~nm}), \sim 2.5 \mathrm{mg}$ (200 $\mathrm{nm}), \sim 5.5 \mathrm{mg}(65 \mathrm{~nm}), \sim 3 \mathrm{mg}(35 \mathrm{~nm}), \sim 2 \mathrm{mg}(25 \mathrm{~nm})$. In octadecylphosphonic acid $\left(\mathrm{C}_{18} \mathrm{H}_{39} \mathrm{O}_{3} \mathrm{P}\right.$; ODPA $)$ modified pores, E7 was spread on the AAO surface modified with (ODPA) at 338 $\mathrm{K}$. The E7 loaded AAO membranes were kept at $343 \mathrm{~K}$ for 24 hours under 200 mbar vacuum. The samples were subsequently quenched with liquid nitrogen, and residual E7 was removed from the AAO surfaces with sharp razor blades and soft polishing paper.

\section{Surface modificaton with ODPA}

Octadecylphosphonic acid was purchased from Alfa Aesar. Selfordered AAO was activated in $30 \%$ aqueous $\mathrm{H}_{2} \mathrm{O}_{2}$ solution for $2 \mathrm{~h}$ at $45{ }^{\circ} \mathrm{C}$, dried at $120{ }^{\circ} \mathrm{C}$ for 15 minutes, and immersed for $48 \mathrm{~h}$ at $25{ }^{\circ} \mathrm{C}$ into a $4.2 \mathrm{mM}$ solution of ODPA in a $n$-heptane/ isopropanol (v:v/5:1) solvent mixture. Subsequently, the substrates were washed with copious amounts of $n$-heptane/ isopropanol mixture and acetone for several times and sonicated to remove any physisorbed (not grafted) ODPA. Finally, they were dried under vacuum (200 mbar) at room temperature overnight. Surface modification with phosphonate coupling agents bearing long alkyl chains (typically from 8 to $18 \mathrm{C}$ atoms) leads to the formation of monolayers.

\section{Surface analysis of ODPA modified AAO membranes}

XPS measurements were used to determine if the surfaces of the pores were successfully coated with the ODPA molecule (Fig. S1, $\mathrm{ESI} \dagger$ ). Thermo Scientific K-Alpha instrument and a $300 \mathrm{~W} \mathrm{MgK \alpha}$ $(1253.6 \mathrm{eV}) \mathrm{X}$-ray source were used for the analysis. The spectrum was taken at an angle of $60^{\circ}$ to the surface.

\section{Differential scanning calorimetry (DSC)}

Thermal analyses were carried out with a Mettler Toledo Star DSC. A 2-12 mg portion of the sample material was placed in aluminum pans that were sealed before the measurements. In the case of bulk E7, empty aluminum pans were used as reference. In the case of AAO infiltrated with E7, native AAO pieces (with the same pore diameter, the same pore depth, and the same pore area) were used as reference samples. Firstly, the sample cooled at a rate of $10 \mathrm{~K} \mathrm{~min}^{-1}$ from room temperature to $173 \mathrm{~K}$ and then heated to $353 \mathrm{~K}$ at the same rate in a nitrogen atmosphere. The same cycle was repeated two times and the results from the second heating and cooling thermographs were employed in the analysis of the phase transition (nematicisotropic) and glass transition temperatures. Temperaturemodulated DSC (TM-DSC) was also employed using a Q2000 (TA Instruments) calorimeter (University of Ioannina). Temperature ramps in the vicinity of the N/I transition were performed at a rate of $2 \mathrm{~K} \mathrm{~min}^{-1}$ with a modulation amplitude $A$ $=0.04 \mathrm{~K}$ and period $T=20 \mathrm{~s}$. This allowed for the extraction of the temperature dependent phase shift, $\delta$, between the applied heating rate (source) and heat flow rate (sample).

\section{Dielectric spectroscopy}

Dielectric measurements were carried out at atmospheric pressure for temperatures $T=215.15-263.15 \mathrm{~K}$ and frequencies $f=5 \times 10^{-2}$ to $1.1 \times 10^{7} \mathrm{~Hz}$ using a Novocontrol Alpha frequency analyzer with an active sample head. The complex dielectric permittivity $\varepsilon^{*}=\varepsilon^{*}(\omega, T, P)$ is a function of frequency $\omega$, temperature $T$, and in general pressure $P$; it is expressed as a sum of real and imaginary parts, $\varepsilon^{*}=\varepsilon^{\prime}-\mathrm{i} \varepsilon^{\prime \prime}$, corresponding to storage and loss of the external electric field's energy, 
respectively. For bulk E7, the DS measurements were carried out in the usual parallel plate geometry with electrodes of $20 \mathrm{~mm}$ in diameter and a sample thickness of $50 \mu \mathrm{m}$ maintained by Teflon spacers. For confined E7, an electrode of $10 \mathrm{~mm}$ in diameter was placed on top of AAO whereas the aluminum bottom of the AAO templates served as the lower electrode. Care was taken to account for the fact that the system under consideration is a heterogeneous dielectric consisting of a mixture of the confining matrix material (AAO) and the LC whose dynamics are of interest (E7). The measured dielectric spectra were corrected for the geometry by using two capacitors in parallel (composed of $\varepsilon_{\mathrm{E} 7}^{*}$ and $\varepsilon_{\mathrm{AAO}}^{*}$ and the measured total impedance was related to the individual values through $\left.1 / Z^{*}=1 / Z_{\mathrm{E} 7}^{*}+1 / Z_{\mathrm{AAO}}^{*}\right)$. In the analysis of DS spectra, the empirical equation of Havriliak and Negami $(\mathrm{HN})^{21}$

$$
\varepsilon_{\mathrm{HN}}^{*}(\omega, \mathrm{T})=\varepsilon_{\infty}(T)+\frac{\Delta \varepsilon(T)}{\left[1+\left(\mathrm{i} \omega \tau_{\mathrm{HN}}(T)\right)^{m}\right]^{n}}+\frac{\sigma_{0}(T)}{\mathrm{i} \varepsilon_{0} \omega}
$$

has been employed. $\tau_{\mathrm{HN}}$ is the characteristic relaxation time, $\Delta \varepsilon$ is the relaxation strength of the process under consideration, $m$, $n$ (with limits $0 \leq m, m n \leq 1$ ) describe, respectively, the symmetric and asymmetric broadening of the relaxation times' distribution, $\sigma_{0}$ is the dc-conductivity and $\varepsilon_{0}$ is the vacuum permittivity. From $\tau_{\mathrm{HN}}$ the relaxation time at maximum loss, $\tau_{\text {max }}$, is obtained analytically as

$$
\tau_{\max }=\tau_{\mathrm{HN}} \sin ^{-1 / m}\left[\frac{\pi m}{2(1+n)}\right] \sin ^{-1 / m}\left[\frac{\pi m n}{2(1+n)}\right]
$$

Both the measured $\varepsilon^{\prime \prime}$ spectra and the derivative of $\varepsilon^{\prime}\left(\mathrm{d} \varepsilon^{\prime} / \mathrm{d} \ln \omega\right.$ $\left.\sim-(2 / \pi) \varepsilon^{\prime \prime}\right)$ have been used in fitting relaxation processes which are visible and hidden by the conductivity contribution, respectively. ${ }^{22}$ In the spectra where more than one relaxation processes contribute to $\varepsilon^{*}$, a summation of $\mathrm{HN}$ functions was employed.

\section{Computational methods}

Molecules 5CB, 7CB, 8OCB and 5CT were fully optimized with no constraints and tight optimization criteria were employed (RMS force criterion was set to $1 \times 10^{-5}$ ). The UltraFine integration grid for numerical integrations was selected (99-point radial quadrature and 590-point Lebedev angular quadrature) to obtain smooth convergence of the floppy parts of the molecules. Frequency calculations confirmed that stationary points are local minima. The alkyl groups for all molecules were initially set to all trans conformation. The combination of the DFT-B3LYP ${ }^{23,24}$ level of theory and cc-pVTZ ${ }^{25}$ basis set were employed which was recently shown to reproduce experimental values of dipole moments with high accuracy. ${ }^{26}$ For all computations Gaussian 16 (ref. 27) software package was employed.

\section{Results and discussion}

\section{Bulk phase study}

The composition of E7 as well as pertinent physical properties of its constituents are summarized in Table 1, from DFT calculations and literature values. ${ }^{28-33}$ The dipole moments of all molecules along with the pictorial representation of their optimized structures are also shown in Table 1. For molecules $5 \mathrm{CB}, 7 \mathrm{CB}$ and $5 \mathrm{CT}$ the vector of the dipole moment is almost parallel to the aromatic rings and the nitrile bond while the values of the dipole moment lie close to each other: 6.1, 6.2, and 6.4 debye respectively. For molecule $80 \mathrm{CB}$ a greater deviation from parallelicity is observed for the vector of the dipole moment while the value of the dipole moment is 7.3 debye. This difference is attributed to the ether group present in 8OCB. ${ }^{31}$ The cartesian coordinates of the optimized structures for all molecules are given in the ESI section. $\dagger$

The thermal properties of bulk E7 are concisely depicted in the thermogram of Fig. 1. Cooling and subsequent heating at a rate of $10 \mathrm{~K} \mathrm{~min}^{-1}$ reveal two distinct features. First, an exo/ endo thermic peak on cooling/heating at the N/I transition (at $\sim 333 \mathrm{~K})$ and, second, a step-like change at the glass temperature (at $\sim 208 \mathrm{~K}$ ) (the minor feature at $\sim 305 \mathrm{~K}$ is a baseline feature). Concerning which element of the mixture influences the phase state most, as a first approximation we consider the weighted average of the constituents' $T_{\mathrm{N} / \mathrm{I}}$ and obtain a trial value of $T_{\mathrm{N} / \mathrm{I}}=333.8 \mathrm{~K}$. Surprisingly, this estimate is quite close to the actual $T_{\mathrm{N} / \mathrm{I}}$. Therefore, we may assume that E7's thermal properties are the composition of those belonging to the individual molecules comprising the mixture. The exceptionally large range of nematic stability can be understood as the inability of the different molecules to form smectic or crystalline phases due to packing constraints imposed by the different length scales of individual components. Furthermore, we may assume that the mixture's molecules retain mobility down to the lowest $T_{\mathrm{g}}$ of the individual components in the mixture. In this case, comparing the observed $T_{\mathrm{g}}$ for $\mathrm{E} 7$ with those of $5 \mathrm{CB}, 7 \mathrm{CB}$ and $8 \mathrm{OCB}$, we conclude that the liquid-to-glass temperature is determined largely by $5 \mathrm{CB}(e . g$. the majority component that is also the more mobile).

Fig. 2 displays the isochronal $\left(f=10^{6} \mathrm{~Hz}\right)$ temperature dependence of the dielectric permittivity, $\varepsilon^{\prime}$, on heating bulk E7 at a rate of $\mathrm{d} T / \mathrm{d} t=5 \mathrm{~K} \mathrm{~min}^{-1}$. The $\mathrm{N} / \mathrm{I}$ transition appears as a discontinuous decrease of the dielectric permittivity at $\sim 330$ K. Upon approaching $T_{\mathrm{N} / \mathrm{I}}$, a substantial portion of the bulk nematic phase $(\sim 15 \mathrm{~K})$ exhibits a gradual reduction of $\varepsilon^{\prime}$ prior to the transition suggesting a large range of pre-transitional fluctuations. Furthermore, within the isotropic phase of bulk E7, we note an almost temperature independent dielectric permittivity. This is contrary to theory which predicts a $T^{-1}$ orientational contribution to the dielectric permittivity as $^{21,22}$

$$
\varepsilon_{\mathrm{s}}^{\prime}-\varepsilon_{\infty}=\frac{1}{3 \varepsilon_{o}} F g \frac{\mu^{2}}{k_{\mathrm{B}} T} \frac{N_{0}}{V}
$$

Here, $F=\varepsilon_{\mathrm{s}}^{\prime}\left(\varepsilon_{\infty}+2\right)^{2} / 3\left(2 \varepsilon_{\mathrm{s}}^{\prime}+\varepsilon_{\infty}\right)$ is the local field, $N_{0} / V$ is the number density of dipoles expressed as $(\rho / M) N_{\mathrm{A}}$, where $\rho$ is the mass density, $M$ is the molar mass and $N_{\mathrm{A}}$ is Avogadro's number, $\mu$ is the dipole moment of a single independent molecule and $g$ is the dipolar orientation correlation function defined according to Kirkwood and Fröhlich as 
Table 1 Constituent molecules of E7 along with their gas phase dipole moment, mass, clearing and glass temperature. $\mu_{\text {gas }}$ is the gas phase (i.e., non-interacting) dipole moment obtained by use of DFT calculations. In the case of E7, the gas phase dipole moment is rudimentarily calculated using a weighted average of the corresponding moments of its constituents

\begin{tabular}{|c|c|c|c|c|}
\hline LC compound & $\mu_{\text {gas }}$ (debye) & Molar mass $\left(\mathrm{g} \mathrm{mol}^{-1}\right)$ & $T_{\mathrm{N} / \mathrm{I}}(\mathrm{K})$ & $T_{\mathrm{g}}(\mathrm{K})$ \\
\hline $5 \mathrm{CB}(51 \%)$ & 6.1 & 249.36 & 308.4 & 210 \\
\hline 7CB $(25 \%)$ & 6.2 & 277.41 & 315.85 & 268 \\
\hline $8 \mathrm{OCB}(16 \%)$ & 7.3 & 307.44 & 353.15 & 232 \\
\hline $5 \mathrm{CT}(8 \%)$ & 6.4 & 325.45 & 513.15 & - \\
\hline
\end{tabular}

$$
g=1+\frac{\left\langle\sum_{i=1}^{N_{0}} \sum_{i<j} \mu_{i} \mu_{j}\right\rangle}{N_{0} \mu^{2}}
$$

From eqn (3) using the values $M=271.75 \mathrm{~g} \mathrm{~mol}^{-1}, \rho=$ $1.03 \mathrm{~g} \mathrm{~cm}^{-3}, \mu=6.3$ debye and $\varepsilon_{\infty}=n^{2}=2.59,{ }^{34}$ we extract a value of $g \sim 0.25$ (inset, Fig. 2). This indicates that the isotropic phase exhibits non-zero dipolar correlations with antiparallel alignment of neighboring dipoles as $g<1$. This is consistent with both previous experimental observations ${ }^{35}$ and simulation results. ${ }^{36}$ A value of $g=1$ would correspond to the case of noncorrelated dipoles, therefore, the fact that even as we progress $40 \mathrm{~K}$ into the isotropic phase the correlation factor has only increased by $6 \%$ towards a hypothetical isotropic value of unity

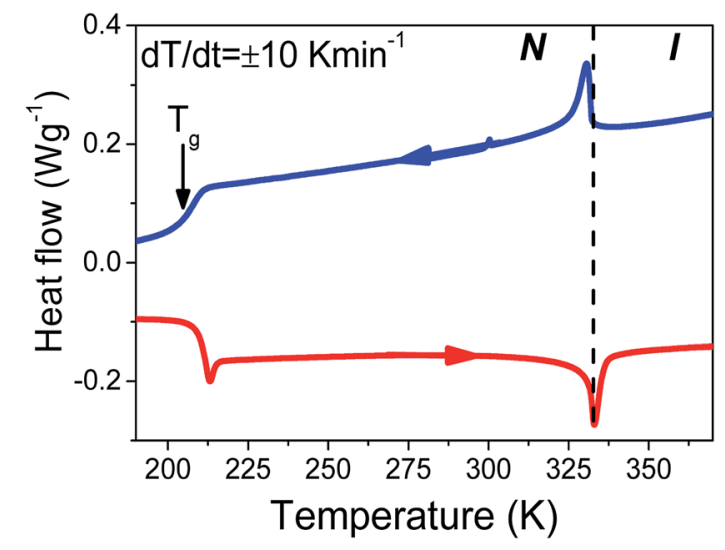

Fig. 1 Thermogram of bulk E7 obtained on cooling and subsequent heating at $10 \mathrm{~K} \mathrm{~min}^{-1}$. Two well separated thermal signatures at $\sim 333 \mathrm{~K}$ and $\sim 208 \mathrm{~K}$ correspond to the $\mathrm{N} / \mathrm{I}$ transition and glass temperature, respectively. The dashed line indicates the nematic to isotropic transition temperature. suggests a persistence of dipolar correlations for a substantial fraction of the isotropic phase.

\section{E7 dynamics in the bulk}

Because of its wide range of nematic stability, E7 lends itself to an exhaustive study of the various relaxation modes of a nematogen. Theoretical models which employ orientational probability distribution functions and take into account only the point symmetry of the nematic phase predict the existence of four dielectrically active relaxation domains..$^{37,38}$ These are associated with molecules aligned either parallel or perpendicular to the probing field performing end-over-end rotation about the short molecular axis (slow) and precession/nutation about the nematic director or rotation about the long molecular axis (fast). Experimental studies on liquid crystalline

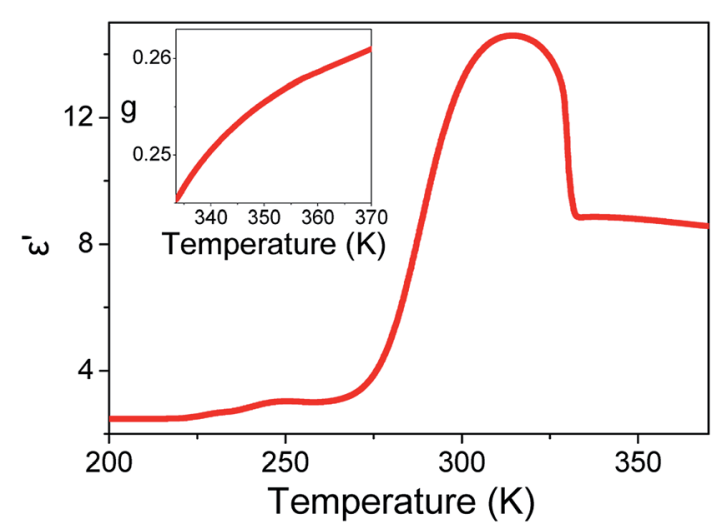

Fig. 2 Isochronal $\left(f=10^{6} \mathrm{~Hz}\right.$ ) temperature dependence of the dielectric permittivity, $\varepsilon^{\prime}$, on heating bulk E7 at a rate of $\mathrm{d} T / \mathrm{d} t=5$ $\mathrm{K} \mathrm{min}^{-1}$. (Inset) Temperature evolution of Kirkwood-Fröhlich dipolar correlation factor in the isotropic phase of bulk E7. A value less than unity indicates persisting anti-parallel dipolar correlations. 
dynamics tend to identify two processes termed $\alpha$-(fast) and $\delta$ (slow) which are well separated within the nematic phase due to the nematic potential which hinders the dynamics of the latter mode..$^{39,40}$ In the present study isothermal loss spectra of bulk E7 exhibit four modes of dielectric relaxation here labeled as $\kappa$, $\lambda, \delta$ and $\alpha$ processes (Fig. 3 ). The $\alpha$ and $\delta$ processes are assigned to molecules aligned parallel to the probing electric field performing precession about the nematic director and reorientation about the short molecular axis, respectively. Similarly, the $\kappa$ and $\lambda$ processes are assigned to the same motions but for molecules aligned almost perpendicularly to the electric field. Previous studies have identified the freezing of the $\alpha$-process as responsible for the LC's glass temperature. ${ }^{9-11} \mathrm{~A}$ faster mode associated with the reorientation about the long molecular axis is not dielectrically active.

The relaxation times for end-over-end rotational motions $(\delta$ and $\kappa$ processes) can be described by a Debye $(m=m n=1)$ distribution. Precessional motions about the director $(\alpha$ and $\lambda$ processes) exhibit a distribution of relaxation times with corresponding $\mathrm{HN}$ parameters $m=0.9, m n=0.5$ and $m=0.75, m n$ $=1$, respectively. As these should be the faster relaxation modes, for molecules parallel and almost perpendicular to the probing field, the fact that they do not exhibit a Debye time distribution is indicative of an underlying distribution of angles between the molecules and the external field or nematic director. ${ }^{31}$ The temperature evolution of all processes in Fig. 3 can be described by a Vogel-Fulcher-Tammann (VFT) dependence as

$$
\tau=\tau_{0} \mathrm{e}^{B /\left(T-T_{0}\right)}
$$

where $T_{0}$ is the process's so called ideal glass temperature for which its characteristic time goes to infinity and $B=f(T)$ is the activation parameter. This allows the determination of the glass temperature for each process by employing the definition of $T_{\mathrm{g}}$ as the temperature where the relaxation time is at $\tau \sim 100 \mathrm{~s}$ (Fig. 4). Note that the dynamics of $\alpha$ - and $\delta$-processes converge to a single $T_{\mathrm{g}} \sim 209 \mathrm{~K}$, quite close to that obtained by DSC, whereas $\lambda$ and $\kappa$ processes freeze at substantially higher temperatures (clearly time-temperature superposition is not valid). Based on this finding we may assume that $\lambda$ and $\kappa$ processes describe cooperative motions which disrupt a large number of neighboring dipoles. Furthermore, we note that in the bulk, the main relaxation process is the $\delta$-process with values of $T \Delta \varepsilon$ larger than those of the $\alpha$-process by factors of 60 to 30 , at higher and lower temperatures, respectively.

\section{Effect of confinement on phase behavior}

The effect of confinement can be discussed by performing isochronal measurements of the dielectric permittivity. Earlier studies have shown the sensitivity of the $\varepsilon^{\prime}(T)$ dependence to various phase transitions and even to the identification of new phases under confinement..$^{\mathbf{2 8 , 3 1 , 4 1}}$ Fig. 5 depicts isochronal $(f=$ $10^{6} \mathrm{~Hz}$ ) measurements of the dielectric permittivity, $\varepsilon^{\prime}$, at a rate of $5 \mathrm{~K} \mathrm{~min}^{-1}$ for bulk and confined $\mathrm{E} 7$ within native and ODPA modified AAO. ODPA adsorbed form organic solvents forms stable monolayers on the amorphous surface of AAO pores resulting in hydrophobic low-energy surfaces. ${ }^{42,43}$ The representation of $\varepsilon^{\prime}$ is supplemented with its absolute derivative with respect to temperature, $\left|\mathrm{d} \varepsilon^{\prime} / \mathrm{d} T\right|$. This allows greater sensitivity in detecting even weak thermodynamic and dynamic transitions as has been shown earlier by some of us.

In regard to the effect of confinement, first we note, upon increasing confinement in both native (Fig. 5(a)) and ODPA modified (Fig. 5(b)) AAO pores, a depression and broadening of $T_{\mathrm{N} / \mathrm{I}}{ }^{44}$ The fact that the step in $\varepsilon^{\prime}\left(T=T_{\mathrm{N} / \mathrm{I}}\right)$ reduces with decreasing pore size is equivalent to the decreasing values of $\left|\mathrm{d} \varepsilon^{\prime} / \mathrm{d} T\right|\left(T=T_{\mathrm{N} / \mathrm{I}}\right)$ and indicates a weakening of the transition in confinement. The decrease of $T_{\mathrm{N} / \mathrm{I}}$ with confinement can be described by a linear dependence versus inverse pore diameter as $T_{\mathrm{m}}=T_{\mathrm{m}}{ }^{\infty}-A / d$ where $T_{\mathrm{m}}{ }^{\infty}=329.8 \pm 0.5 \mathrm{~K}$ and $A=77 \pm$ $32 \mathrm{~nm}$. This is discussed considering a simplistic model of a nematic in contact with a solid substrate. The effect of the solid substrate is to introduce a perturbation of the nematic ordering, with lengthscales comparable to the molecular length, creating an interface or even an interphase. The presence of adsorbed molecules leads to an increase in surface tension whereas the fixed mean orientation of molecules at the
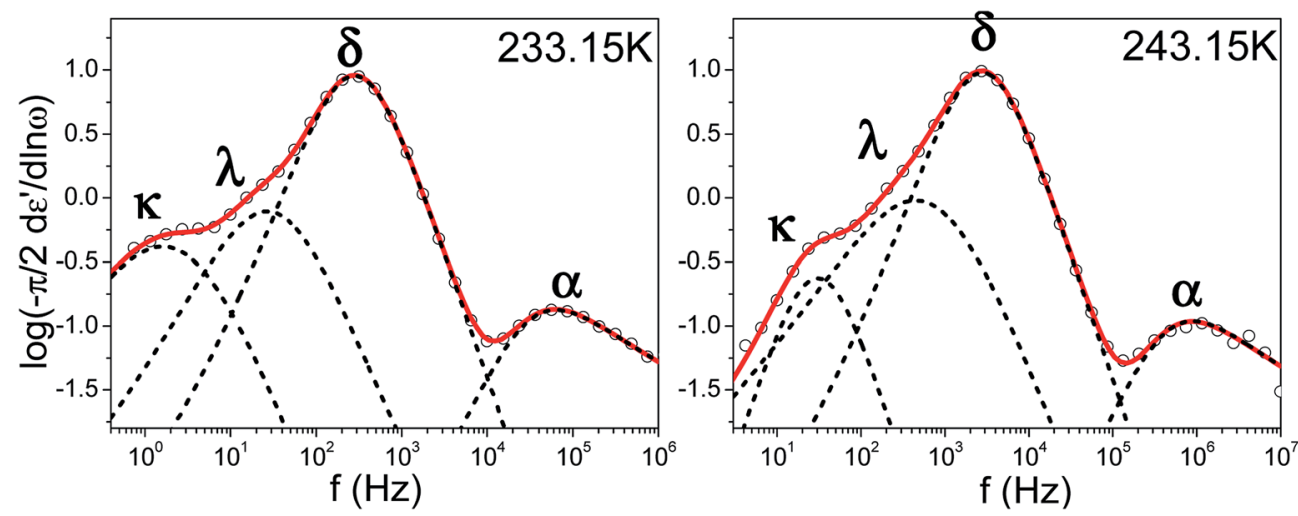

Fig. 3 Characteristic isothermal loss spectra of bulk E7 for two temperatures well within the nematic phase; (left) $T=233.15 \mathrm{~K}$ (right) $T=243.15$ $K$. Circles correspond to experimental data and red curves to fits performed using a summation of Havriliak-Negami functions, dashed black peaks confirm the applicability of deconvolution of the loss spectra into four dielectrically active modes labeled as $\kappa, \lambda, \delta, \alpha$ processes. 


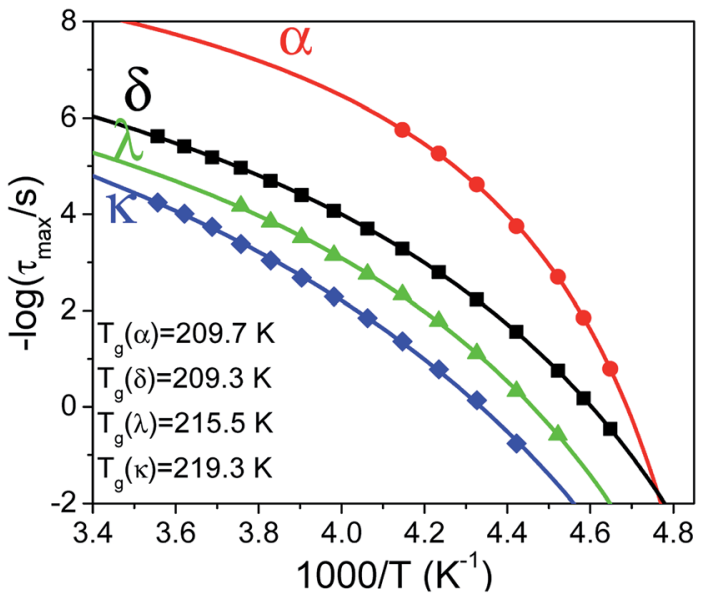

Fig. 4 Temperature evolution of all relaxation modes present in bulk E7; symbols correspond to modes as follows, (red circles) $\alpha$-process, (black squares) $\delta$-process, (green triangles) $\lambda$ process, (blue rhombi) $\kappa$ process. Solid curves indicate each process's fits with a VFT dependence allowing the extraction of its respective $T_{\mathrm{g}}$. Note that $\alpha$-and $\delta$ processes exhibit a $T_{\mathrm{g}}$ similar to that observed by DSC.

interface (surface anchoring) affects molecules farther away from the surface due to anchoring; molecules arrange in such a conformation that reduces the elastic energy but deforms the equilibrium director field. Therefore, at a given temperature
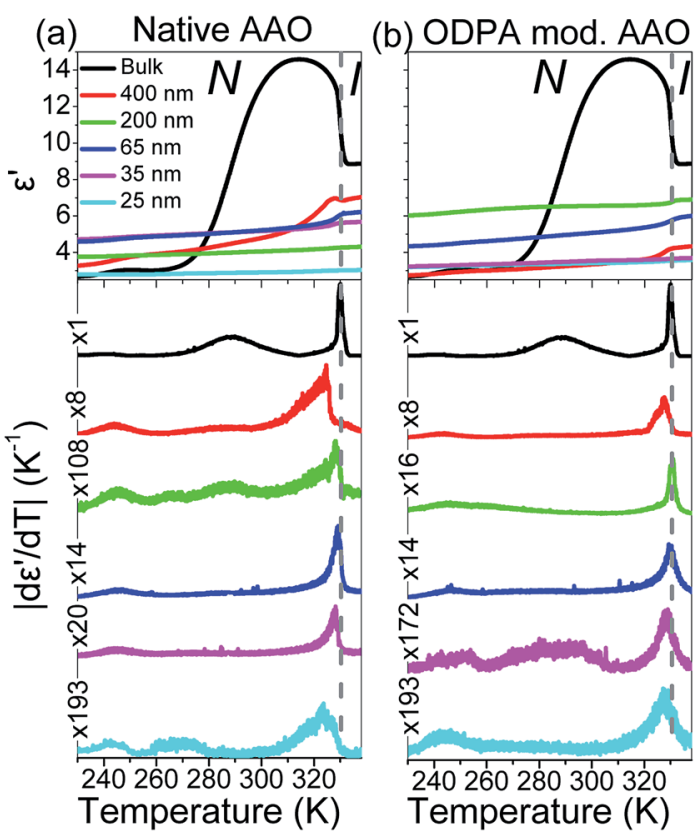

Fig. 5 Comparison of the dielectric permittivity and its absolute derivative with temperature for bulk E7 and E7 confined within native (a) and ODPA modified (b) AAO pores measured at $f=10^{6} \mathrm{~Hz}$ and dT/ $\mathrm{d} t=5 \mathrm{~K} \mathrm{~min}^{-1}$. Notice the necessary magnification factors applied to the vertical axes of confined curves suggesting a weaker $\mathrm{N} / \mathrm{I}$ transition in confinement. Signatures at $T<T_{N / l}$ are not of thermodynamic origin and correspond to resonances of dynamic processes at specific temperatures for the given frequency. (i.e., only spatially dependent director field) the free energy density includes contributions from elastic (volume) and surface terms. ${ }^{45-47}$ These two contributions yield a decrease in $T_{\mathrm{N} / \mathrm{I}}$ which is expressed analytically as

$$
\Delta T=\frac{\frac{k}{S^{2}}}{2 a_{0}}\left(\frac{2 \pi}{R}\right)^{2}
$$

where $k$ is a Frank elastic constant in the nematic liquid crystal phase, $S$ is the scalar nematic order parameter, $a_{0}$ is the Landaude Gennes free energy expansion constant and $R$ the pore radius, and

$$
\Delta T=\frac{2 T_{\mathrm{N} / \mathrm{I}} \Delta \sigma}{R \Delta H \rho}
$$

Here, $\Delta \sigma$, is the difference in surface tension between the nematic and isotropic phases, $\Delta H$ is the enthalpy associated with the transition and $\rho$ the density of the liquid crystal. From eqn (6) considering twist $\left(k_{22}=4.5 \times 10^{-12} \mathrm{~N}\right)$ and bend $\left(k_{33}=\right.$ $13 \times 10^{-12} \mathrm{~N}$ ) distortions ${ }^{48}$ separately, a typical value of $S=0.3$ in the nematic phase and $a_{0}=9 \times 10^{4} \mathrm{~J} \mathrm{~m}^{-3} \mathrm{~K}$ (ref. 46) yields depressions equal to $0.27 \mathrm{~K}(R=400 \mathrm{~nm}), 70 \mathrm{~K}(R=25 \mathrm{~nm})$ and $0.79 \mathrm{~K}(d=400 \mathrm{~nm}), 200 \mathrm{~K}(d=25 \mathrm{~nm})$, respectively. From eqn (7) using values $T_{\mathrm{N} / \mathrm{I}}=333.79 \mathrm{~K}, \Delta \sigma=0.026 \times 10^{-3} \mathrm{~N} \mathrm{~m}^{-1},{ }^{46} \Delta H$ $=4.78 \mathrm{~J} \mathrm{~g}^{-1}$ and $\rho=1.03 \mathrm{~g} \mathrm{~cm}^{-3}$, we obtain depressions equal to $0.017 \mathrm{~K}(d=400 \mathrm{~nm})$ and $0.28 \mathrm{~K}(d=25 \mathrm{~nm})$. Representative experimental values of $\Delta T$ are equal to $5.5 \mathrm{~K}$ and $6.8 \mathrm{~K}$ in native $400 \mathrm{~nm}$ and $25 \mathrm{~nm}$ pores, respectively. We may conclude that the depression of $T_{\mathrm{N} / \mathrm{I}}$ in E7 contains contributions from both elastic distortions and surface tension effects.

Secondly, comparing the form of $\left|\mathrm{d} \varepsilon^{\prime} / \mathrm{d} T\right|\left(T=T_{\mathrm{N} / \mathrm{I}}\right)$ in native and ODPA modified AAO, the latter exhibit a broader and more symmetrical shape than the former. This can be understood in light of orientation effects; ODPA induces homeotropic anchoring of molecules at the pore's surface creating a spatially dependent director field. Therefore, material in different parts of the pore is subject to varying boundary conditions, such as surface pinning or intermolecular elastic forces, requiring a larger range of temperatures to undergo the transition. This is consistent with an earlier study of cyanobiphenyls confined to $200 \mathrm{~nm}$ Anopore membranes ${ }^{4}$ where $C_{\mathrm{p}}\left(T=T_{\mathrm{N} / \mathrm{I}}\right)$ peaks exhibited greater depression, broadening and symmetry in radial rather than axial alignment.

Lastly, apart from the N/I transition, we observe low temperature signatures in the $\left|\mathrm{d} \varepsilon^{\prime} / \mathrm{d} T\right|$ curves of all samples. These are attributed to resonances of dipolar relaxation (i.e., loss) processes which exhibit maximum losses at a certain temperature when examined at a constant frequency of the external electric field and are subsequently discussed.

\section{Effect of confinement on dynamics}

More informative of the dynamic processes exhibited in confined E7 are the isothermal loss spectra in Fig. 6. All confined samples, within native AAO pores, exhibit $\alpha$ and $\delta$ processes; $\lambda$ and $\kappa$ processes are both seen in $400 \mathrm{~nm}$ pores with the former persisting in 65 and $25 \mathrm{~nm}$ pores. In ODPA 
modified pores, $\alpha$ - and $\delta$-processes are evident in addition to the $\kappa$ process in $400 \mathrm{~nm}$ and $200 \mathrm{~nm}$ pores and the $\lambda$ process in $65 \mathrm{~nm}$ and $25 \mathrm{~nm}$ pores. The existence of at least one $\kappa$ or $\lambda$ process is indicative of non-perfect nematic alignment even within the confining matrices. In addition, all spectral modes display broadening of relaxation time distributions with increasing confinement.

In fact, the broadening of $\alpha$ - and $\delta$-relaxation time distributions is almost identical for diameters of a specific pore size, irrespective of surface properties, with an exception in the case of $25 \mathrm{~nm}$ pores where the low frequency wing of the $\delta$-process is wider than its native counterpart as $m_{\text {native }}=0.85$ and $m_{\mathrm{ODPA}}=$ 0.48. However, already from the low temperature regions in Fig. 5 and from Fig. 6, we observe that E7 confined to ODPA modified pores exhibits a persistent preference for relaxation via the $\alpha$-process. Oppositely, within native AAO pores, the preferred mode is the $\delta$-process in $400 \mathrm{~nm}$ and $25 \mathrm{~nm}$ pores versus the $\alpha$-process in $65 \mathrm{~nm}$ pores. Therefore, it is presumed that molecular alignment in native $400 \mathrm{~nm}$ and $25 \mathrm{~nm}$ pores is axial whereas in $65 \mathrm{~nm}$ pores it is planar radial or escaped radial. A similar observation was made in the case of $5 \mathrm{CB}^{23}$ where confinement in native $35 \mathrm{~nm}$ and $25 \mathrm{~nm}$ pores yielded a preference for $\alpha$ - or $\delta$-dynamics, respectively, concluding that the selection of dynamics in confinement is influenced by size effects which are also in competition with surface induced orientation effects.

We observe that for all confined samples the ratios of $T \Delta \varepsilon(\delta) /$ $T \Delta \varepsilon(\alpha)$ are much smaller than that in the bulk, with values of 1.53, 0.27 and 1.17 in native pores with sizes of $400 \mathrm{~nm}, 65 \mathrm{~nm}$ and $25 \mathrm{~nm}$, respectively, and values $0.32,0.28,0.2,0.6,0.6$ in treated pores with respective sizes of $400 \mathrm{~nm}, 200 \mathrm{~nm}, 65 \mathrm{~nm}$, $35 \mathrm{~nm}$ and $25 \mathrm{~nm}$. As seen in Fig. 6, this can be attributed to a decrease of $\Delta \varepsilon(\delta)$ indicating that slow modes are generally less favored in confinement than in the bulk.

Both $\alpha$ - and $\delta$-modes in confinement exhibit a VFT temperature dependence with similar relaxation times for each separate process in native and modified confinement (Fig. 7).
Corresponding fits are performed using a constant $\tau_{0}$ for each process equal to that acquired from the bulk $\left(-\log \tau_{0}(\alpha)=10.4\right.$ and $\left.-\log \tau_{0}(\delta)=9.7\right)$. This arises from the assumption that at the high temperature limit (i.e., well within the isotropic phase) dynamics should be unaffected by confinement. The values of VFT fitting parameters obtained for $\alpha$ - and $\delta$-processes within native and ODPA modified pores are listed in Tables 2 and 3, respectively. We have also included, for both processes and types of pores, the values of the isobaric steepness index defined as $^{49}$

$$
m^{*}=\left[\mathrm{d}(\log \tau) / \mathrm{d}\left(T_{\mathrm{g}} / T\right)\right]_{T_{\mathrm{g}}}
$$

Comparing the parameters from Tables 2 and 3 we observe that fits yield, in all diameters irrespective of surface properties, higher values of the $B$ parameter for the $\delta$-process relative to the $\alpha$-process, suggestive of the higher energy cost of activating a cooperative motion. Furthermore, in both types of pores we note an increase in fragility, or equivalently a decrease in the steepness index, of the $\alpha$-process and a concomitant increase in that of the $\delta$-process with confinement. Lastly, for a given diameter, the fragility corresponding to the $\alpha$-process in native pores is somewhat higher than that in ODPA modified pores. This suggests that, a reduction of temperature, even close to the $T_{\mathrm{g}}$, affects the dynamics associated with the $\alpha$-process more in ODPA modified pores than in native pores. Extracting the glass temperature of the $\alpha$-process in both types of confining pores, we note a depression with decreasing pore size described as $T_{\mathrm{g}}(\alpha)=T_{\mathrm{g}}^{\mathrm{bulk}}(\alpha)-A / d$ with $T_{\mathrm{g}}^{\text {bulk }}(\alpha)=209.8 \pm 0.3 \mathrm{~K}$ and $A=75 \pm$ $15 \mathrm{~nm}$; a similar, though not as strong depression is seen for the glass temperature of the $\delta$-process with $T_{\mathrm{g}}^{\mathrm{bulk}}(\delta)=209.9 \pm 0.2 \mathrm{~K}$ and $A=19 \pm 12 \mathrm{~nm}$. The reduction in both $T_{\mathrm{N} / \mathrm{I}}$ and $T_{\mathrm{g}}(\alpha), T_{\mathrm{g}}(\delta)$ results in a downward shift of the nematic phase upon reducing pore diameter. This is concisely depicted in the corresponding phase diagram (Fig. 8). The steeper $T_{\mathrm{g}}(\alpha$ or $\delta$ ) as compared to the $T_{\mathrm{N} / \mathrm{I}}$ dependence on inverse pore diameter effectively enlarges the $\mathrm{N}$ phase region under confinement.
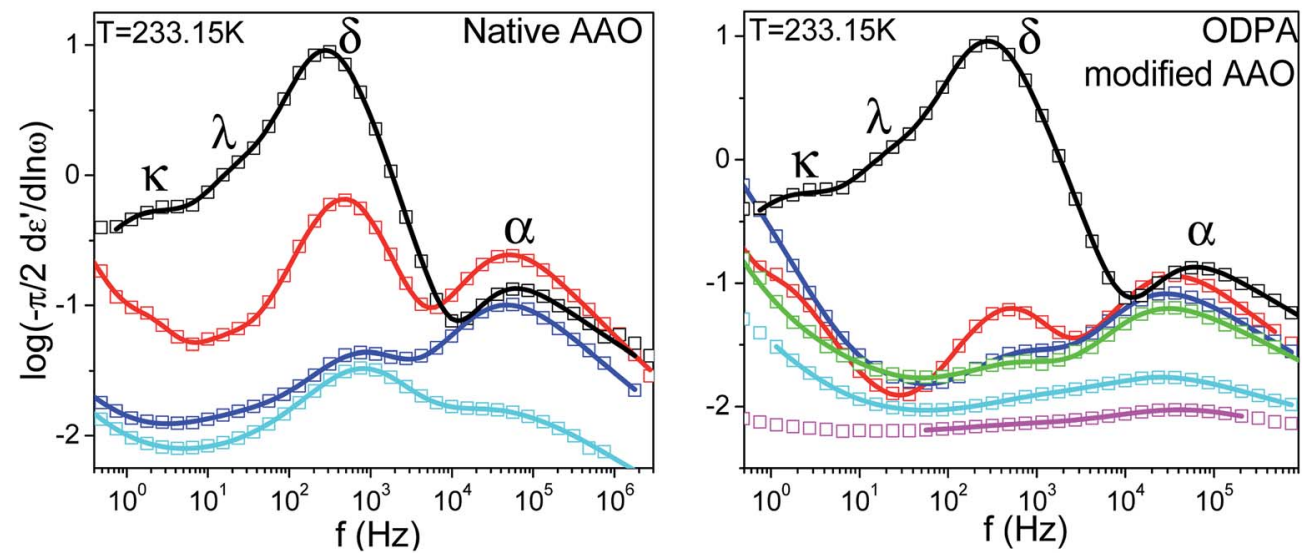

Fig. 6 Dielectric loss spectra of bulk E7 and E7 confined to native (left) and ODPA modified (right) AAO. Open symbols correspond to experimental data and solid curves to fits using a summation of Havriliak-Negami functions. Varying diameters are depicted as follows, (black) bulk E7; (red) $400 \mathrm{~nm}$; (green) $200 \mathrm{~nm}$; (blue) $65 \mathrm{~nm}$; (magenta) $35 \mathrm{~nm}$; (cyan) $25 \mathrm{~nm}$. Note that in native confinement either $\alpha$ - or $\delta$-dynamics could be preferred whereas when confinement is superimposed with surface treatment a constant preference for $\alpha$-dynamics is exhibited. 

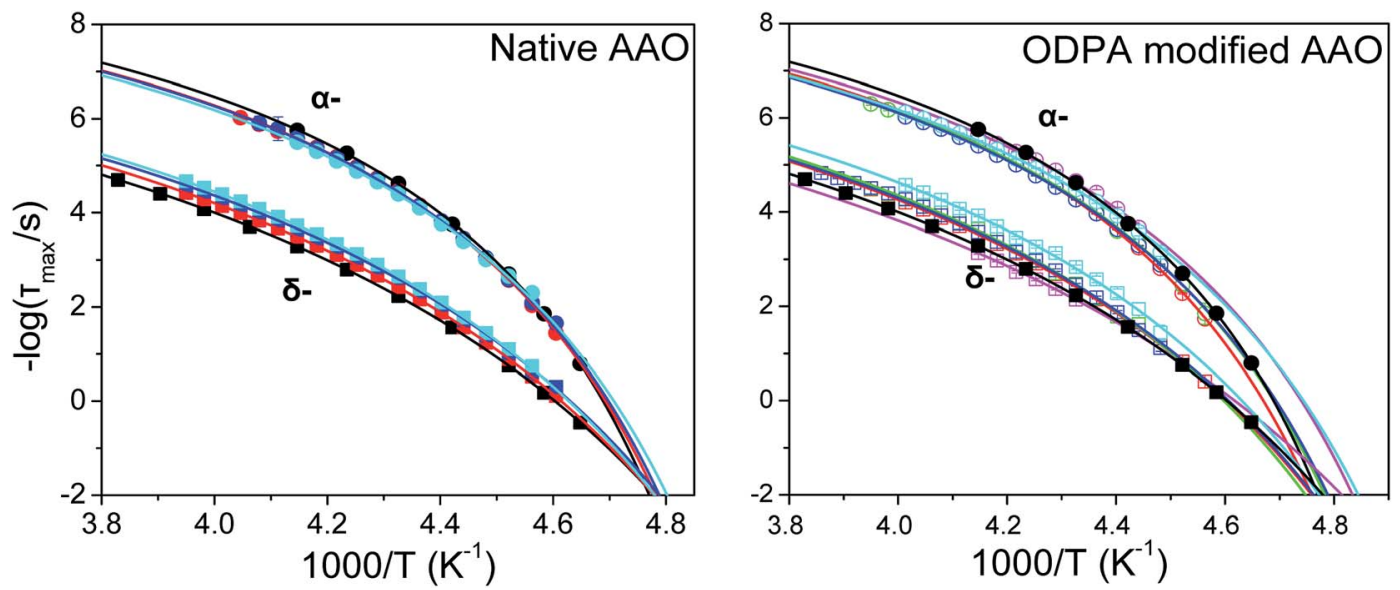

Fig. 7 Temperature dependence of $\alpha$ and $\delta$-relaxation times in bulk E7 and E7 located within native AAO (left) and ODPA modified AAO (right). Both exhibit a VFT dependence and, most notably reduction of $T_{\mathrm{g}}(\alpha)$ and $T_{\mathrm{g}}(\delta)$ with decreasing pore diameter. Varying diameters are depicted as follows, (black) bulk E7; (red) $400 \mathrm{~nm}$; (green) $200 \mathrm{~nm}$; (blue) $65 \mathrm{~nm}$; (magenta) $35 \mathrm{~nm}$; (cyan) $25 \mathrm{~nm}$.

The enhanced mobility that results from the decrease in the glass temperature can be discussed within the framework of the rotational diffusion model. ${ }^{50}$ The latter considers a diffusing rod-like molecule confined in a conical region in which all orientations up to a maximum cone angle are equally probable. The calculated dipolar autocorrelation function is found to decay rapidly as the conical volume (or angle) decreases. Therefore, an accelerated rotation of low molecular weight mesogens is predicted as they are confined to a decreasing volume.

\section{Effect of confinement on the nature of the N/I transition}

E7 confined to native and ODPA treated pores shows strong preand meta-transitional phenomena in the vicinity of the $\mathrm{N} / \mathrm{I}$ transition, evident in the absolute derivative of the dielectric permittivity. Treated pores, in particular, exhibited not only broadening but also an increasingly symmetric form upon increasing confinement. They are, therefore, ideal in examining the effect of confinement on the pre- and meta-transitional fluctuations around the N/I transition. The following equations $^{51}$ were used to describe the "critical" behavior of the quantity $\left|\mathrm{d} \varepsilon^{\prime} / \mathrm{d} T\right|$ in the vicinity of the N/I transition:

$$
\left|\frac{\mathrm{d} \varepsilon^{\prime}}{\mathrm{d} T}\right|_{\mathrm{L}}=A+B_{\mathrm{L}}\left|\frac{T-T^{* * *}}{T^{* *}}\right|^{-\alpha}+C\left[\frac{T-T^{* *}}{T^{* *}}\right]
$$

$$
\left|\frac{\mathrm{d} \varepsilon^{\prime}}{\mathrm{d} T}\right|_{\mathrm{H}}=A+B_{\mathrm{H}}\left|\frac{T-T^{*}}{T^{*}}\right|^{-\alpha}+C\left[\frac{T-T^{*}}{T^{*}}\right]
$$

According to eqn (9) and (10), approaching the transition from below and above, respectively, physical quantities exhibit an exponential divergence versus the so called "reduced temperature". ${ }^{52,53}$ Reduced temperature is, on either side of the transition, a measure of distance from the virtual transition temperatures $T^{*}, T^{* *}$, i.e., the temperature at which the transition would occur if it were continuous. $T^{*}$ corresponds to the limit of supercooling the isotropic phase (obtained by extrapolating the fluctuations above the transition) and $T^{* *}$ to the limit of superheating the nematic phase (obtained by extrapolating the fluctuations below the transition). The existence of a nonzero metastability region, $\Delta T=T^{* *}-T^{*}$, is indicative of a first-order transition whereas a second-order or continuous transition is characterized by $\Delta T=0 .{ }^{54}$ In eqn (9) and (10) the terms $B_{\mathrm{L}}\left|T-T^{* *} / T^{* *}\right|^{-\alpha}, B_{\mathrm{H}}\left|T-T^{*} / T^{*}\right|^{-\alpha}$ are different on either side of the peak while $A, C$ are kept equal on either side of the peak constituting the non-singular background. Fits were performed simultaneously on both sides of the $\left|\mathrm{d} \varepsilon^{\prime} / \mathrm{d} T\right|$ peaks corresponding to the N/I transition (in ODPA modified AAO pores) in ranges of $10 \mathrm{~K}$ into nematic and isotropic phases. A common coexistence range of $3.6 \mathrm{~K}$ about the peak maximum

Table 2 Values of the activation parameter, ideal glass temperature, glass temperature $(\tau \sim 100 \mathrm{~s})$ and fragility index obtained from VFT fits of $\alpha$ -

\begin{tabular}{|c|c|c|c|c|c|c|c|c|}
\hline Native AAO & $B(\mathrm{~K})$ & $T_{\infty}(\mathrm{K})$ & $T_{\mathrm{g}}(\mathrm{K})$ & $m^{*}$ & $B(\mathrm{~K})$ & $T_{\infty}(\mathrm{K})$ & $T_{\mathrm{g}}(\mathrm{K})$ & $m^{*}$ \\
\hline $400 \mathrm{~nm}$ & $590 \pm 3$ & $188.5 \pm 0.2$ & $209.2 \pm 0.2$ & 126 & $976 \pm 1$ & $173.1 \pm 0.1$ & $209.3 \pm 0.1$ & 67 \\
\hline $65 \mathrm{~nm}$ & $597 \pm 2$ & $188.0 \pm 0.2$ & $208.9 \pm 0.2$ & 124 & $932 \pm 3$ & $174.3 \pm 0.2$ & $208.9 \pm 0.2$ & 70 \\
\hline $25 \mathrm{~nm}$ & $625 \pm 5$ & $186.4 \pm 0.4$ & $208.3 \pm 0.4$ & 118 & $898 \pm 1$ & $175.9 \pm 0.1$ & $209.2 \pm 0.1$ & 73 \\
\hline
\end{tabular}
and $\delta$-relaxations for $E 7$ in native AAO pores 
Table 3 Values of the activation parameter, ideal glass temperature, glass temperature $(\tau \sim 100 \mathrm{~s})$ and fragility index obtained from VFT fits of $\alpha$ and $\delta$-relaxations for E7 in ODPA modified AAO pores

\begin{tabular}{|c|c|c|c|c|c|c|c|c|}
\hline \multirow{2}{*}{$\begin{array}{l}\text { ODPA modified } \\
\text { AAO }\end{array}$} & \multicolumn{4}{|l|}{$\alpha$-process } & \multicolumn{4}{|l|}{$\delta$-process } \\
\hline & $B(\mathrm{~K})$ & $T_{\infty}(\mathrm{K})$ & $T_{\mathrm{g}}(\mathrm{K})$ & $m^{*}$ & $B(\mathrm{~K})$ & $T_{\infty}(\mathrm{K})$ & $T_{\mathrm{g}}(\mathrm{K})$ & $m^{*}$ \\
\hline $400 \mathrm{~nm}$ & $599 \pm 4$ & $189.2 \pm 0.3$ & $210.2 \pm 0.3$ & 124 & $936 \pm 1$ & $175.42 \pm 0.1$ & $210.2 \pm 0.1$ & 71 \\
\hline $200 \mathrm{~nm}$ & $620 \pm 6$ & $187.5 \pm 0.4$ & $209.2 \pm 0.4$ & 119 & $896 \pm 13$ & $177.4 \pm 0.8$ & $210.9 \pm 0.9$ & 73 \\
\hline $65 \mathrm{~nm}$ & $633 \pm 2$ & $186.7 \pm 0.1$ & $208.8 \pm 0.2$ & 117 & $928 \pm 7$ & $175.6 \pm 0.5$ & $210.0 \pm 0.5$ & 72 \\
\hline
\end{tabular}

was excluded in order to consider the fluctuations pertinent to each pure phase; this region was extracted from the divergence of the phase shift recorded from TM-DSC on heating at a rate of $5 \mathrm{~K} \mathrm{~min}^{-1}$ using a temperature amplitude $A=0.04 \mathrm{~K}$ and period $T=20$ s. Proximity of the N/I transition to a tricritical point ${ }^{12,55,56}$ was assumed by using $\alpha=0.5$. This allowed for estimation of a region of metastability, $\Delta T$, about the actual transition equal to $T^{* *}-T^{*}$. The extracted metastability region's, $\Delta T=T^{* *}-T^{*}$ width, shows a systematic reduction with increasing confinement (Fig. 9) indicating a weakening of the N/I transition. However, as even in the severest examined confinement (e.g. 25 $\mathrm{nm}), \Delta T \neq 0$, we conclude that the transition is not yet continuous.

Plotting the width of $\Delta T$ versus inverse pore diameter allows for the estimation of a hypothetical critical diameter at which the N/I transition becomes continuous (i.e., $\Delta T=0$ ). Due to the sudden decrease in $\Delta T$ when going from bulk to confined conditions, two linear fits are performed and this is shown in

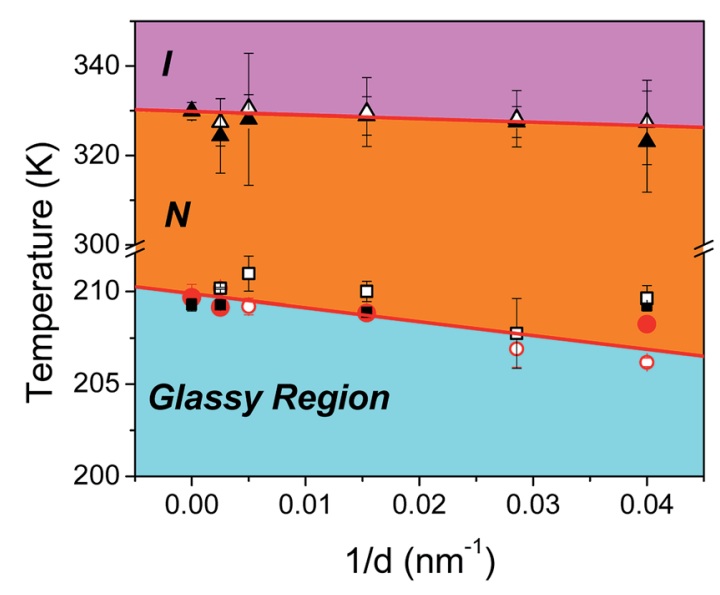

Fig. 8 Effective phase diagram describing the phase state and dynamics of confined E7. Filled (open) triangles correspond to $T_{\mathrm{N} / \mathrm{l}}$ as obtained from isochronal DS measurements in bulk and native (modified) AAO pores. Filled (open) red circles correspond to $T_{g}(\alpha)$ in the bulk and native (modified) AAO pores; filled (open) black squares correspond to $T_{\mathrm{g}}(\delta)$ in the bulk and native (modified) AAO pores. Linear fits are shown as red lines where the freezing of the $\alpha$-process has been used as the upper limit of the glassy region. The effect of confinement is to shift the nematic region to lower temperatures and to accelerate dynamics with decreasing pore size.
Fig. 10. Depending on the method of extrapolation the extracted critical diameter ranges from $11 \mathrm{~nm}$ to $23 \mathrm{~nm}$. This is similar to the critical diameters previously estimated ( $23 \mathrm{~nm}$ and $16 \mathrm{~nm})$ from cyanobiphenyls $7 \mathrm{CB}$ and $8 \mathrm{CB}$, respectively, imbibed in tubular silica nanochannels. ${ }^{57}$

\section{Origin of the N/I transition in a mixture system}

In the preceding discussion we have considered two independent quantities as markers of the N/I transition's strength: initially, the value of $\left|\mathrm{d} \varepsilon^{\prime} / \mathrm{d} T\right|\left(T=T_{\mathrm{N} / \mathrm{I}}\right)$ and, subsequently, the width of the metastability region $\Delta T$ about the actual transition. As a non-zero value of both quantities is related to the first-order

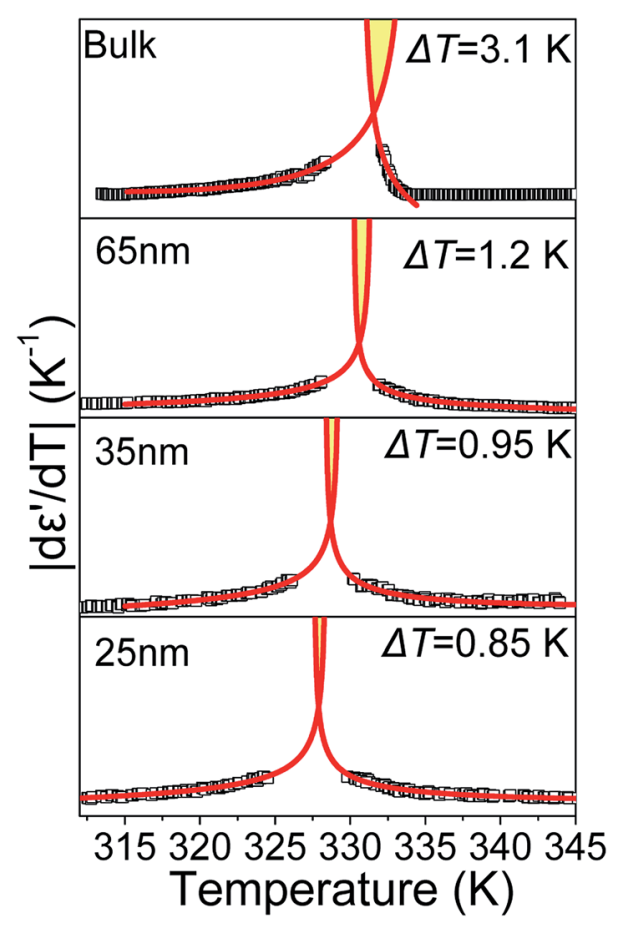

Fig. 9 Isochronal $\left|d \varepsilon^{\prime} / d T\right|$ peaks corresponding to the $N / I$ transition in bulk E7 and E7 confined to 65, 35 and $25 \mathrm{~nm}$ AAO. Extrapolation of temperature dependent fluctuations yields a region of metastability, indicative of the transition's strength, which decreases with confinement. Data points within the two-phase coexistence region are not shown in order to depict only the fluctuations pertinent to the N/I transition. 


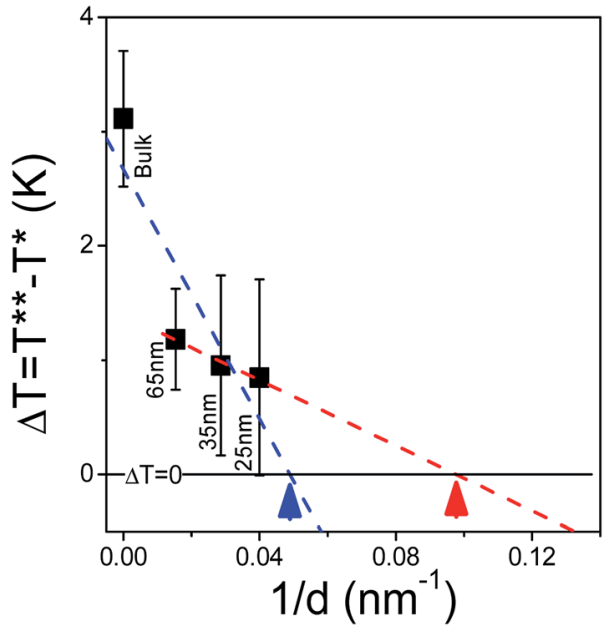

Fig. 10 The decrease of the metastability region associated with the $\mathrm{N} / \mathrm{I}$ transition is extrapolated in order to estimate the critical diameter at which the transition becomes continuous yielding an upper limit (blue dashed line and blue arrow) of $23 \mathrm{~nm}$ and a lower limit (red dashed line and corresponding red arrow) of $11 \mathrm{~nm}$.

character of the N/I transition it is reasonable to expect a linear correspondence between the two. This is shown in Fig. 11. To this end we consider the ratio $\left|\mathrm{d} \varepsilon^{\prime} / \mathrm{d} T\right|\left(T=T_{\mathrm{N} / \mathrm{I}}\right) / \Delta T$ extracted as the slope of the plot $\left|\mathrm{d} \varepsilon^{\prime} / \mathrm{d} T\right|\left(T=T_{\mathrm{N} / \mathrm{I}}\right)=f(\Delta T)$. The value of the aforementioned slope is calculated for E7 and compared to those of conventional thermotropic LCs belonging the cyanobiphenyl group, 8CB and 8OCB and studied earlier by us. ${ }^{31}$

Fits include the point $(0,0)$ as a value of zero for $\left|\mathrm{d} \varepsilon^{\prime} / \mathrm{d} T\right|(T=$ $\left.T_{\mathrm{N} / \mathrm{I}}\right)$ would imply the same for $\Delta T$, and vice versa. The extracted slopes are $(0.34 \pm 0.09)$ for $8 \mathrm{OCB},(0.17 \pm 0.02)$ for $8 \mathrm{CB}$ and $(0.05$ \pm 0.02 ) for E7. This is interpreted as an inherently weaker N/I

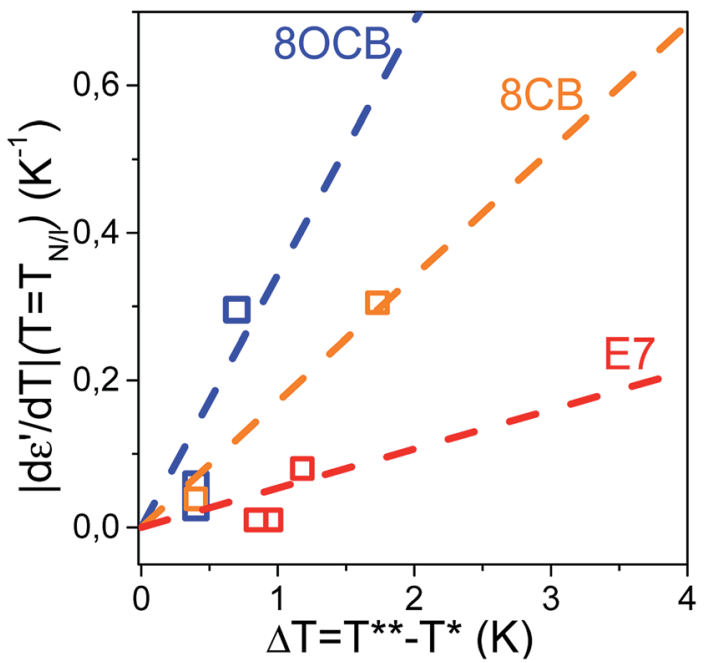

Fig. 11 Linear fits of the plot $\left|d \varepsilon^{\prime} / d T\right|\left(T=T_{N / 1}\right)=f(\Delta T)$ for pure systems (8CB, 8OCB) and the mixture system E7 reveal a significantly smaller slope of the latter. This indicates a weakening effect of mixing on the $\mathrm{N} / \mathrm{I}$ transition. transition in the mixture system, E7. We extend our argument by noticing that for all LCs in confinement the slope of $\left|\mathrm{d} \varepsilon^{\prime} / \mathrm{d} T\right|$ $\left(T=T_{\mathrm{N} / \mathrm{I}}\right)=f(\Delta T)$ is less than unity. Therefore, in a confined length scale the first-order nature of a transition is more pronounced in the thermal fluctuations about the transition temperature than the sudden divergence at exactly the transition temperature; this indicates the increasing potential of fluctuations in destabilizing ordered phases in confinement.

Central to the understanding of the origin of the N/I in the eutectic mixture E7 is a comparison with its constituents. Fig. 12 compares $\varepsilon^{\prime}(T)$ for $\mathrm{E} 7,8 \mathrm{CB}, 8 \mathrm{OCB}$ and $5 \mathrm{CB}$ in a range of about $\pm 4 \mathrm{~K}$ about their respective $T_{\mathrm{N} / \mathrm{I}}$ 's. Note the sharp, steplike, decrease for pure systems at $T_{\mathrm{N} / \mathrm{I}}$ in contrast to E7 which exhibits an S-shape decrease. In the $\left|\mathrm{d} \varepsilon^{\prime} / \mathrm{d} T\right|$ representation this is portrayed as a broader peak for E7 with strong pre- and metatransitional fluctuations (inset to Fig. 12). This can be explained as follows; in pure systems, pre-transitional phenomena are attributed to order parameter fluctuations, with the increase in thermal energy, which eventually lead to an order-disorder transition at $T_{\mathrm{N} / \mathrm{I}}$. However, in E7 we note a large temperature range of fluctuations which must be attributed not only to pure thermal disordering but, also, to local concentration fluctuations triggered by the different constituents of the mixture exhibiting different length scales and ranges of nematic stability.

It must be stressed that 5CB and $80 \mathrm{OCB}$ account for $67 \%$ of $\mathrm{E} 7$, therefore the gradual character of the transition in E7 is not inherent in its constituents but results due to the effect of mixing. This leads to the conclusion that the mixing of LCs renders the final system eutectic with a single, widened nematic range at the cost of an equally protracted and gradual transition.

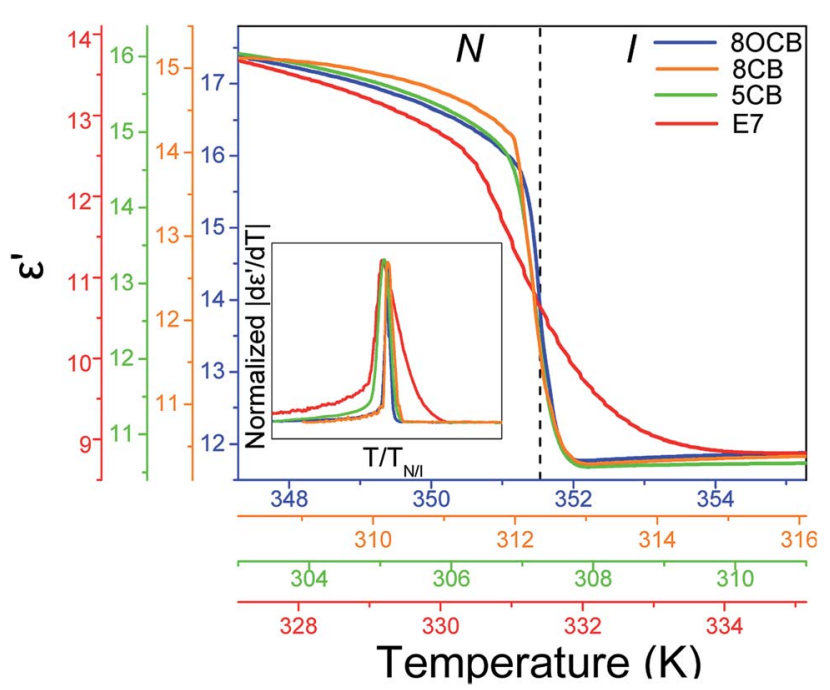

Fig. 12 Measurements of the dielectric permittivity on heating through the $\mathrm{N} / \mathrm{l}$ transition in bulk $80 \mathrm{CB}$ (blue), $8 \mathrm{CB}$ (orange), 5CB (green) and E7 (red) (inset) $\left|\mathrm{d} \varepsilon^{\prime} / \mathrm{d} T\right|$ curves for bulk $80 \mathrm{CB}$ (blue), 5CB (green), E7 (red) normalized with their respective peak heights and transition temperatures. The S-shape of $\varepsilon^{\prime}$ for E7 (or, equivalently, the broader form of $\left.\left|d \varepsilon^{\prime} / d T\right|\right)$ indicates a gradual transition with order fluctuations contributing over a widened temperature range. 


\section{Conclusions}

Two-dimensional hard confinement of the eutectic compound E7 in self-ordered AAO membranes alters its phase behavior, molecular dynamics and nature of the N/I transition. Decreasing pore size resulted in a depression of $T_{\mathrm{N} / \mathrm{I}}$ due to the effect of surface tension and elastic distortions on the nematic order. Dynamics were found to accelerate with increasing confinement, as predicted by the rotational diffusion model, resulting in a decreasing $T_{\mathrm{g}}$. This resulted in displacing the region of nematic stability towards lower temperatures with decreasing pore diameter. The effect of surface treatment was seen as a preference for relaxation via the least cooperative $\alpha$ mode due to constraints imposed by homeotropic anchoring at the pore surface. Confinement was found to weaken the N/I transition as evidenced by a reduction in the corresponding metastability region's width from $3.1 \mathrm{~K}$ to $0.85 \mathrm{~K}$. Extrapolation of this weakening to a zero-valued metastability region allowed the calculation of a critical diameter (in the range from $23 \mathrm{~nm}$ to $11 \mathrm{~nm}$ ) at which the transition is expected to become continuous. Lastly, comparing the N/I transition in the E7 to those of pure systems by use of the proposed ratio $\left|\mathrm{d} \varepsilon^{\prime} / \mathrm{d} T\right|\left(T=T_{\mathrm{N} / \mathrm{I}}\right) / \Delta T$ revealed a broader transition in the former. This was interpreted as the result of local concentration fluctuations - triggered by the different lengthscales and nematic ranges of the components - on top of the conventional thermally activated order fluctuations.

These results are not only of technological relevance in the implementation of devices with tunable dielectric properties but also in the understanding of the physics of phase transitions in soft matter under confinement.

\section{Conflicts of interest}

There are no conflicts to declare.

\section{Acknowledgements}

The computations in this paper were run on the Odyssey cluster supported by the FAS Division of Science, Research Computing Group at Harvard University. We thank Professor Kaxiras for providing access to its cluster. H. D. gratefully acknowledges the Max-Planck-Gesellschaft (MPG) for financial support of the MPIP-TOBB ETU Partner Group Program. AAO infiltration process support by E. Tuncel is gratefully acknowledged.

\section{References}

1 P. G. de Gennes, and J. Prost, The Physics of Liquid Crystals, Oxford University Press, Oxford (UK), 1994.

2 G. P. Crawford, and S. Zumer, Liquid crystals in complex geometries, Taylor and Francis, London, 1996.

3 G. S. Iannacchione and D. Finotello, Phys. Rev. Lett., 1992, 69, 2094.

4 G. S. Iannacchione and D. Finotello, Phys. Rev. E, 1994, 50, 4780.
5 S. A. Rozanski, R. Stannarius, H. Groothues and F. Kremer, Liq. Cryst., 1996, 20, 59.

6 S. Diez, M. A. Perez-Jubindo, M. R. de la Fuente, D. O. López, J. Salud and J. L. Tamarit, Liq. Cryst., 2006, 9, 1083.

7 Y. Trukhina and T. Schilling, Phys. Rev. E, 2008, 77, 011701.

8 G. P. Crawford, R. Ondris-Crawford, S. Žumer and J. W. Doane, Phys. Rev. Lett., 1993, 70, 1838.

9 A. R. Brás, H. Dionísio and A. Schönhals, J. Phys. Chem. B, 2008, 112, 8227.

10 M. Mierzwa, M. Paluch, S. J. Rzoska, J. Zioło, and U. Maschke, Soft Matter under Exogenic Impacts, Springer, 2007, pp. 201-214.

11 A. Schönhals, S. Frunza, L. Frunza, T. Unhruh, B. Frick and R. Zorn, Eur. Phys. J.: Spec. Top., 2010, 189, 251.

12 V. M. Lenart, S. L. Gómez, I. H. Bechtold, A. M. Figueiredo Neto and S. R. Salinas, Eur. Phys. J. E, 2012, 35, 4.

13 M. C. W. van Boxtel, M. Wübbenhorst, J. van Turnhout, C. W. M. Bastiaansen and D. J. Broer, Liq. Cryst., 2003, 30(2), 235.

14 M. T. Viciosa, A. M. Nunes, A. Fernandes, P. L. Almeida, M. H. Godinho and M. D. Dionísio, Liq. Cryst., 2002, 29, 429.

15 A. R. Brás, M. Dionísio and A. Schönhals, J. Phys. Chem. B, 2008, 112, 8227.

16 A. R. Brás, S. Frunza, L. Guerreiro, I. M. Fonseca, A. Corma, L. Frunza, M. Dionísio and A. Schönhals, J. Chem. Phys., 2010, 132, 224508.

17 H. Masuda and K. Fukuda, Science, 1995, 268, 1466-1468.

18 H. Masuda, F. Hasegwa and S. Ono, J. Electrochem. Soc., 1997, 144, L127.

19 H. Masuda, K. Yada and A. Osaka, Jpn. J. Appl. Phys., 1998, 37, L1340.

20 M. Steinhart, Adv. Polym. Sci., 2008, 220, 123.

21 F. Kremer, and A. Schönhals, in Broadband Dielectric Spectroscopy, Springer, Berlin, 2002.

22 G. Floudas, Polymer Science: A Comprehensive Reference, in Dielectric Spectroscopy, ed. K. Matyjaszewski, and M. Möller, Elsevier BV, Amsterdam, vol. 2.32, pp.825-845, 2012.

23 A. D. Becke, J. Chem. Phys., 1993, 98, 5648.

24 C. Lee, W. Yang and R. G. Parr, Phys. Rev. B: Condens. Matter Mater. Phys., 1988, 37, 785.

25 T. H. Dunning, J. Chem. Phys., 1989, 90, 1007.

26 A. L. Hickey and C. N. Rowley, J. Phys. Chem. A, 2014, 118, 3678.

27 M. J. Frisch, G. W. Trucks, H. B. Schlegel, G. E. Scuseria, M. A. Robb, J. R. Cheeseman, G. Scalmani, V. Barone, G. A. Petersson, H. Nakatsuji, X. Li, M. Caricato, A. V. Marenich, J. Bloino, B. G. Janesko, R. Gomperts, B. Mennucci, H. P. Hratchian, J. V. Ortiz, A. F. Izmaylov, J. L. Sonnenberg, D. Williams-Young, F. Ding, F. Lipparini, F. Egidi, J. Goings, B. Peng, A. Petrone, T. Henderson, D. Ranasinghe, V. G. Zakrzewski, J. Gao, N. Rega, G. Zheng, W. Liang, M. Hada, M. Ehara, K. Toyota, R. Fukuda, J. Hasegawa, M. Ishida, T. Nakajima, Y. Honda, O. Kitao, H. Nakai, T. Vreven, K. Throssell, J. A. Montgomery Jr, J. E. Peralta, F. Ogliaro, M. J. Bearpark, J. J. Heyd, E. N. Brothers, K. N. Kudin, V. N. Staroverov, T. A. Keith, R. Kobayashi, J. Normand, 
K. Raghavachari, A. P. Rendell, J. C. Burant, S. S. Iyengar, J. Tomasi, M. Cossi, J. M. Millam, M. Klene, C. Adamo, R. Cammi, J. W. Ochterski, R. L. Martin, K. Morokuma, O. Farkas, J. B. Foresman, and D. J. Fox, Gaussian 16, Revision B.01, Gaussian, Inc., Wallingford CT, 2016.

28 C. Grigoriadis, H. Duran, M. Steinhart, H.-J. Butt and G. Floudas, ACS Nano, 2011, 5, 9208-9215.

29 A. Hourri, T. K. Bose and J. Thoen, Phys. Rev. E, 2001, 63, 051702.

30 K. C. Sungh and P. C. Jain, Struct. Chem., 1996, 7, 215.

31 A. Selevou, G. Papamokos, M. Steinhart and G. Floudas, J. Phys. Chem. B, 2017, 121, 7382.

32 J. Jiang, E. Zhuravlev, Z. Huang, L. Wei, Q. Xu, M. Shan, G. Xue, D. Zhou, C. Schick and W. Jiang, Soft Matter, 2013, 9, 1488-1491.

33 B. Lamia, N. Gogibus, B. Ewen, T. Pakula, X. Coqueret, M. Benmouna and U. Maschke, Polymer, 2004, 45, 6555.

34 C.-S. Yang, C.-J. Lin, R.-P. Pan, C. T. Que, K. Yamamoto, M. Tani and C.-L. Pan, J. Opt. Soc. Am. B, 2010, 27, 1866.

35 Y. Kim, M. Lee, H. S. Wang, S. Ahn and J. Kim, Vib. Spectrosc., 2017, 92, 182.

36 J. Peláez and M. Wilson, Phys. Chem. Chem. Phys., 2007, 9, 2968.

37 A. Kozak, J. K. Moscicki and G. Williams, Mol. Cryst. Liq. Cryst., 1991, 201, 1-12.

38 S. Diez, M. A. Perez-Jubindo, M. R. de la Fuente, D. O. López, J. Salud and J. L. Tamarit, Liq. Cryst., 2006, 9, 1083.

39 G. Meier and A. Saupe, Mol. Cryst., 1966, 1, 515-525.

40 A. J. Martin, G. Meier and A. Saupe, Symp. Faraday Soc., 1971, 5, 119.

41 H. Duran, H. B. Hartmann-Azanza, M. Steinhart, D. Gehrig, F. Laquai, X. Feng, K. Müllen, H.-J. Butt and G. Floudas, ACS Nano, 2012, 6, 9359.
42 P. Thissen, M. Valtiner and G. Grundmeier, Langmuir, 2010, 26, 156.

43 E. Hoque, J. A. DeRose, G. Kulik, P. Hoffmann, H. J. Mathieu and B. Bhushan, J. Phys. Chem. B, 2006, 110, 10855.

44 D. Wilms, A. Winkler, P. Virnau and K. Binder, Phys. Rev. Lett., 2010, 105, 045701.

45 B. Jérôme, Rep. Prog. Phys., 1991, 54, 391.

46 M. Kuzma and M. Labes, Mol. Cryst. Liq. Cryst., 1983, 100, 103-110.

47 M. Alcoutlabi and G. B. McKenna, J. Phys.: Condens. Matter, $2005,17,461$.

48 B. Klus, U. A. Laudyn, M. A. Karpierz and B. Sahraoui, Opt. Express, 2014, 22, 24.

49 G. Floudas, M. Paluch, A. Grzybowski, and K. Ngai, Molecular dynamics of glass-forming systems, in Effects of pressure, Springer, 2011.

50 C. C. Wang and R. Pecora, J. Chem. Phys., 1980, 72, 5333.

51 S. Diez-Berart, D. O. López, M. R. de la Fuente, J. Salud, M. A. Pérez-Jubindo and D. Finotello, Liq. Cryst., 2010, 37, 893.

52 C. Domb, and M. S. Green, in Phase Transitions and Critical Phenomena, Academic Press, 1972.

53 H. E. Stanley, in Introduction to Phase Transitions and Critical Phenomena, Clarendon Press, Oxford, 1971.

54 M. Kleman, and O. D. Lavrentovich, in Soft Matter Physics: An Introduction, Springer, 2003.

55 P. K. Mukherjee and T. B. Mukherjee, Phys. Rev. B: Condens. Matter Mater. Phys., 1995, 52, 9965.

56 P. K. Mukherjee, J. Phys.: Condens. Matter, 1998, 10, 9191.

57 A. V. Kityk, M. Wolff, K. Knorr, D. Morineau, R. Lefort and P. Huber, Phys. Rev. Lett., 2008, 101, 187801. 